\title{
Lymphocyte Accumulation during Pseudomonas aeruginosa-induced Pneumonia in Rodents Does Not Require CD11a and Intercellular Adhesion Molecule-1
}

\author{
Jennifer L. Wiebke, William M. Quinlan, Nicholas A. Doyle, James E. Sligh, C. Wayne Smith,
} and Claire M. Doerschuk

Section of Pediatric Pulmonology, Department of Pediatrics and the HB Wells Center for Pediatric Research, Indiana University, Indianapolis, Indiana, and the Departments of Molecular and Human Genetics and Pediatrics, Baylor College of Medicine, Houston, Texas

\begin{abstract}
During Pseudomonas aeruginosa-induced pneumonia in rodents, the acute infiltrate of neutrophils is followed by accumulation of lymphocytes in the perivascular connective tissue. The roles of the adhesion molecules CD1 la/CD18 and intercellular adhesion molecule-1 (ICAM-1) in this accumulation of lymphocytes were investigated. The numbers of lymphocytes in $P$. aeruginosa-induced pneumonia were compared in animals treated with blocking antibodies to either CD1 la, ICAM-1, IgG, or no antibody. In other experiments, the lymphocyte accumulation during $P$. aeruginosa-induced pneumonia in ICAM-1 mutant mice was compared with that in wild-type mice. In rats, both a murine anti-rat CD1 la antibody and nonspecific murine IgG partially inhibited the lymphocyte accumulation by 30 to $40 \%$ compared with animals that received no antibodies. In mice, blocking antibodies to either CD1 la or ICAM-1 did not decrease the lymphocyte accumulation compared with mice given IgG or no antibody. Further, there was no attenuation of the lymphocyte accumulation induced by $P$. aeruginosa in the ICAM-1 mutant mice compared with wild-type mice, either in the total number of lymphocytes or the number of $\mathrm{CD} 4^{+}, \mathrm{CD} 8^{+}$, or B cells. We conclude that neither CD11a/CD18 nor ICAM-1 are required for lymphocyte accumulation during $P$. aeruginosa-induced pneumonia in rodents. The partial inhibition of the lymphocyte accumulation in both the anti-CD1la- and IgG-treated rats may be due to nonspecific effects of foreign proteins on cellular functions.
\end{abstract}

There are well-defined lymphocyte compartments in the normal lung, including the pulmonary microvasculature, the hilar and peritracheal lymph nodes, submucosal collections of lymphocytes along the conducting airways, and the alveolar wall (1-3). During normal immune surveillance, lymphocytes "home" to the lung lymphoid tissue by the process of lymphocyte recirculation, the organ-selective trafficking of lymphocytes between lymphoid tissues and peripheral blood via the lymphatics (4). This process relies on constitutively expressed, tissue-specific adhesion molecules and mediates efficient immune surveillance in the organism.

In contrast to the constitutive lymphocyte recirculation that occurs normally in the noninflamed state, lymphocyte

(Received in original form August 12, 1994 and in revised form December 2, 1994)

Address correspondence to: Jennifer L. Wiebke, M.D., James Whitcomb Riley Hospital for Children, 702 Barnhill Dr., Room 2750, Indiana University, Indianapolis, IN 46202-5225.

Abbreviations: 2,4-dinitrofluorobenzene, DNFB; intercellular adhesion molecule-1, ICAM-1; vascular cell adhesion molecule-1, VCAM-1; very late activation antigen-4, VLA-4.

Am. J. Respir. Cell Mol, Biol. Vol. 12. pp. 513-519, 1995 accumulation and activation occurs at sites of inflammation. Lymphocytes play a major role in immune responses by providing antigen specificity and exerting a number of effector functions, including antibody production, cytokine release, and cytotoxicity. The localization of lymphocytes to sites of antigen deposition is required for the expression of a pulmonary immune response.

The recruitment of lymphocytes to sites of inflammation is mediated in part through increased adhesion of the lymphocytes to the endothelium of the inflamed tissue followed by migration into the tissue (5). In this process, soluble mediators released in response to the tissue injury activate or up-regulate the adhesion molecules on both lymphocytes and endothelium. One adhesion system involved in lymphocyte recruitment is mediated through interactions between CD1 1a/CD18, an integrin expressed on lymphocytes, and intercellular adhesion molecule (ICAM)-1 or -2 , endothelial cell ligands $(6,7)$.

To investigate lymphocyte responses in the lung during inflammation, we have examined the pulmonary immune response to Pseudomonas aeruginosa. This organism is a common nosocomial and community-acquired pathogen that causes significant respiratory morbidity. $P$. aeruginosa- 
induced pneumonia in rodents has been used to study microbial virulence factors and host defense mechanisms (8, 9). Histologic examination of the rodent lungs has shown lesions similar to those found in humans with nonbacteremic, chronic $P$. aeruginosa-induced pneumonia, including bronchiolitis, bronchiectasis, and inflammatory cell infiltrates in the lung parenchyma. During $P$. aeruginosa-induced pneumonia in rodents, there is an initial inflammatory infiltrate consisting of neutrophils, followed by a chronic inflammatory and immune response including perivascular lymphocytic infiltration (10).

These studies investigated the roles of the integrin $\mathrm{CD} 1 \mathrm{la} / \mathrm{CD} 18$ and its endothelial ligand, ICAM-1, in the accumulation of lymphocytes within the lung during $P$. aeruginosa-induced pneumonia in rodents. The function of the specific adhesion molecules was investigated in vivo using blocking antibodies to CD1 la and ICAM-1, as well as ICAM-1 mutant mice.

\section{Materials and Methods}

\section{Animals}

Male Lewis white rats, weighing 200 to $250 \mathrm{~g}$, were purchased from Harlan Bioproducts for Science (Indianapolis, IN). Mice of $129 / \mathrm{Sv}$ and C57BL/6J mixed background, with a mutation in ICAM-1 induced by homologous recombinant techniques (11), and mice of the same genetic background weighing 16 to $27 \mathrm{~g}$ were studied.

\section{Reagents}

A mucoid strain of $P$. aeruginosa from the sputum culture of a patient with cystic fibrosis was obtained from the Indiana University Medical Center microbiology laboratory and grown on trypticase soy agar plates (Becton-Dickinson, San Jose, CA).

Antibodies used for in vivo studies included WT.1, a blocking murine monoclonal $\mathrm{IgG} 2 \mathrm{a}$ antibody against rat CDI la; YN1/1.7, a blocking rat monoclonal IgG2b antibody against murine lymphocyte activation antigen (MALA-2) (12), the homolog of human ICAM-1 (kindly provided by Dr. Fumio Takei, University of British Columbia, Vancouver); and KBA, a blocking rat monoclonal IgG2a antibody against mouse CDIla (Serotec, Kidlington, United Kingdom). These reagents were sterile and free of $\mathrm{NaN}_{3}$. Murine IgG and rat IgG (Sigma Chemical Co., St. Louis, MO) were used as control antibodies. Primary antibodies used for immunohistochemistry to detect murine lymphocyte subtypes included $\mathrm{KT} 174$, a rat monoclonal IgG2c antibody against CD4 antigen in mice (Serotec); KT15, a rat IgG2a antibody against CD8a in mice (Serotec); and YE2/36HLK, a rat monoclonal IgG2a antibody against human HLA class II-DR that recognizes all murine B cells (Serotec).

\section{Induction of Pneumonia}

$P$. aeruginos $a$ was suspended in saline at an absorbance of $0.80 \mathrm{~A}$ (rats) or $0.03 \mathrm{~A}$ (mice) as measured by spectrophotometry. These doses of $P$. aeruginosa correspond to approximately $10^{8}$ and $10^{6} \mathrm{cfu} / \mathrm{ml}$, respectively. Preliminary experiments determined that these doses were optimal for the development of lymphocytic infiltrates in the lung parenchyma without significant mortality. After administration of ketamine $(80 \mathrm{mg} / \mathrm{kg}$ intramuscularly) and acepromazine maleate ( 5 to $8 \mathrm{mg} / \mathrm{kg}$ intramuscularly), small skin incisions over the ventral tracheae were made and the tracheae were cannulated with 18-gauge (rat) or 20-gauge (mouse) catheters. Suspensions of $P$. aeruginosa or saline alone, containing colloidal carbon to mark the distribution in the lung, were instilled into the airway via the catheters in rats $(0.1$ $\mathrm{ml} / \mathrm{rat}$ ), ICAM-1 mutant mice, and wild-type mice of the same genetic background $(0.02 \mathrm{ml} / \mathrm{mouse})$. The lungs were removed 7 days later and processed as described subsequently.

\section{Experimental Protocol}

Rats given airway instillation of $P$. aeruginosa received daily tail vein injections of $2 \mathrm{mg} / \mathrm{kg}$ of either the anti-CD1la antibody WT.1 or murine IgG on days 3 through 6 after airway instillation of bacteria. A third group of rats with pneumonia received no antibody. A fourth group of rats given sterile, intrabronchial instillation of saline received no antibody.

Wild-type mice with $P$. aeruginosa-induced pneumonia received either no antibody or daily tail vein injections of $2 \mathrm{mg} / \mathrm{kg}$ of either the anti-CD1 la antibody KBA, the antiICAM-1 antibody YN1/1.7, or rat IgG on days 3 through 6 after intratracheal instillation of $P$. aeruginosa. ICAM-1 mutant mice with pneumonia received no antibody. On day 7 , the animals were anesthetized, blood samples were collected from the inferior vena cava of each animal, and the lungs were removed and fixed as described previously.

\section{Immunochemistry}

To confirm that the anti-CD1 la antibody was present in the bloodstream for at least $24 \mathrm{~h}$ after intravenous injection, serum samples were obtained from the WT.1-treated rats on days 4 through 7, immediately before the daily injection of antibody. Immunochemistry was performed on cytospins of peripheral blood from control rats that had received no antibody, using the serum samples or WT.1 as the primary antibody. Murine IgG was used as a negative control primary antibody. After a 15-min incubation in normal goat serum to block nonspecific background staining, the cytospins were incubated with serum or WT.1 for $30 \mathrm{~min}$. The samples were next incubated with a biotinylated anti-mouse IgG secondary antibody (Kirkegard and Perry, Gaithersburg, MD) for 30 $\mathrm{min}$ and then an alkaline phosphatase-streptavidin conjugate for $30 \mathrm{~min}$ followed by the addition of an alkaline phosphatase substrate that couples with new fuchsin to give a bright red reaction product at the site of enzyme activity.

\section{Determination of Blood Lymphocyte Counts}

To evaluate the effect of blocking CD11a or ICAM-1 function on the number of circulating lymphocytes, blood samples were collected from wild-type mice with $P$. aeruginosa-induced pneumonia that had received daily doses of antiCD1 1a, ICAM-1, or IgG. After lysis of red blood cells with acetic acid, total white blood cell counts were determined by hemocytometer for each sample. The percentages of lymphocytes (identified by characteristic morphology) in the blood samples were determined by cell differentials on hematoxylin-stained blood smears. 


\section{Morphometric Evaluation of Lung Sections}

Accumulation of lymphocytes was quantitated by measuring the density of the lymphocytes in the perivascular regions. On day 7 of $P$. aeruginosa-induced pneumonia, the lungs were removed and fixed by inflation with $4 \%$ glutaraldehyde. Four-micrometer-thick, paraffin-embedded sections from the lungs were stained with hematoxylin and eosin and evaluated with a $40 \times$ oil immersion objective for a final magnification of $400 \times$ using a Nikon Labophot- 2 microscope equipped with a camera lucida attachment and digitizing pad (Jandel Scientific, San Rafael, CA), allowing precise measurement of distances. The inner and outer circumferences were determined by measuring the lengths of the endothelial cells and the outer edge of the perivascular connective tissue, respectively, and the area of the perivascular connective tissue was determined using a digitizer interfaced with software (Sigmascan software; Jandel Scientific). Lymphocytes were identified by characteristic morphology, and the number of lymphocytes in the perivascular region was counted. The lymphocyte density was expressed as the number of lymphocytes per $100 \mu \mathrm{m}^{2}$ of perivascular connective tissue. For each animal, five to eight vessels were examined and the mean lymphocyte density for each animal in each treatment group was then calculated.

\section{Subtypes of Accumulated Lymphocytes}

The subtypes of the accumulated lymphocytes during pneumonia in ICAM-1 mutant and wild-type mice were determined by immunohistochemistry. Lungs from ICAM-1 mutant mice $(n=5)$ and wild-type mice $(n=4)$ were removed on day 7 of $P$. aeruginosa-induced pneumonia, inflated with Tissue-Tek (Miles, Inc., Elkhart, IN), and frozen in liquid $\mathrm{N}_{2}$. Eight-micrometer-thick frozen tissue sections were cut on a cryostat microtome and stored at $-20^{\circ} \mathrm{C}$ until immunohistochemistry was done. After a 10-min fixation in acetone, endogenous biotin was blocked in the tissue sections by incubations with $0.01 \%$ avidin (Sigma) for $15 \mathrm{~min}$, then $0.001 \%$ biotin (Sigma) for $15 \mathrm{~min}$ to saturate all biotin-binding sites on avidin. Nonspecific binding of the secondary antibody was blocked by a 15-min incubation with normal goat serum. The tissue sections were next incubated for 30 min with the primary antibody against either murine $\mathrm{CD}^{+}{ }^{+}$cells, $\mathrm{CD}^{+}$ cells, or B cells, followed by a 30-min incubation with the secondary antibody, a mouse serum-adsorbed goat anti-rat IgG (Kirkegard and Perry), and then a 30-min incubation with streptavidin-alkaline phosphatase complex (Kirkegard and Perry). A 20-min incubation with alkaline phosphatase substrate with new fuchsin was used to detect the positive cells. For each animal, three to five perivascular infiltrates were evaluated. The total number of accumulated lymphocytes in each infiltrate was counted and divided into the number of lymphocytes that stained positively for each primary antibody to obtain the percentage of lymphocytes in the infiltrates that were $\mathrm{CD}^{+}{ }^{+}$cells, $\mathrm{CD}^{+}$cells, and $\mathrm{B}$ cells.

\section{Statistical Analysis}

The mean perivascular lymphocyte densities were compared for all treatment groups. In addition, the vessels were grouped by size to compare the mean lymphocyte densities of the perivascular infiltrates surrounding vessels with inner lumenal areas of $<1,000 \mu \mathrm{m}^{2}$, between 1,000 and 2,000 $\mu \mathrm{m}^{2}$, or $>2,000 \mu \mathrm{m}^{2}$. The effect of inhibiting either CDl la or ICAM-1 on these accumulations was examined by analyzing the lymphocyte density for each group of animals using a one-way ANOVA with a Bonferroni correction (Systat, Evanston, IL) for multiple pairwise comparisons. The statistical analyses of the results of immunochemistry and the determination of blood lymphocyte counts were performed using the unpaired $t$ test. $P<0.05$ was considered significant in all data analyses.

\section{Results}

\section{Accumulation of Lymphocytes in $P$. aeruginosa-induced Pneumonia}

The lungs of rats and mice that received an airway instillation of $P$. aeruginosa showed histologic changes of chronic pneumonia on day 7 compared with the lungs of saline-treated animals (Figure 1). Lymphocytic infiltrates had developed around the pulmonary vasculature, and there were few neutrophils in the alveolar spaces. Areas of the lung that did not receive the bacteria mixed with colloidal carbon did not show any accumulation of lymphocytes.

\section{Role of CD11a in Rats}

Rats that received $P$. aeruginosa showed an 11-fold increase in the number of lymphocytes per $100 \mu \mathrm{m}^{2}$ of perivascular connective tissue compared with animals that received only saline (Figure 2). This increase in lymphocyte density was inhibited by $34 \%$ when animals were treated with an antiCD11a antibody. However, a similar inhibition was seen when the animals were treated with murine IgG. Although both the anti-CDI la-treated and murine IgG-treated animals showed a significant decrease in lymphocyte density compared with rats that received no antibody treatment, no significant differences between the anti-CD1la-treated and murine IgG-treated groups were observed.

The data were also analyzed by comparing the lymphocyte densities in vessels grouped by size (Figure 3). The perivascular infiltrates around vessels $<2,000 \mu \mathrm{m}^{2}$ in crosssectional,area did not show significant differences in lymphocyte densities between any of the groups. The lymphocyte accumulations around vessels $>2,000 \mu \mathrm{m}^{2}$ were significantly smaller in animals treated with the anti-CD1la antibody compared with rats that received no antibody. However, the rats treated with murine IgG also showed reduction in the accumulation of lymphocytes around these vessels similar to the anti-CDIla-treated rats.

\section{Circulation of Anti-CD11a in Rats}

The results of immunochemistry on normal rat cytospins of peripheral blood leukocytes using serum samples, the WT.1 antibody, and murine IgG as primary antibodies are shown in Table 1 . The percentage of positively stained lymphocytes on the cytospins was comparable when either the serum samples or the WT.1 antibody at a concentration of 0.02 $\mathrm{mg} / \mathrm{ml}$ were used as the primary antibody. These results indicate that the injected antibodies remained in the bloodstream and were available to block the CD1 la molecule. 


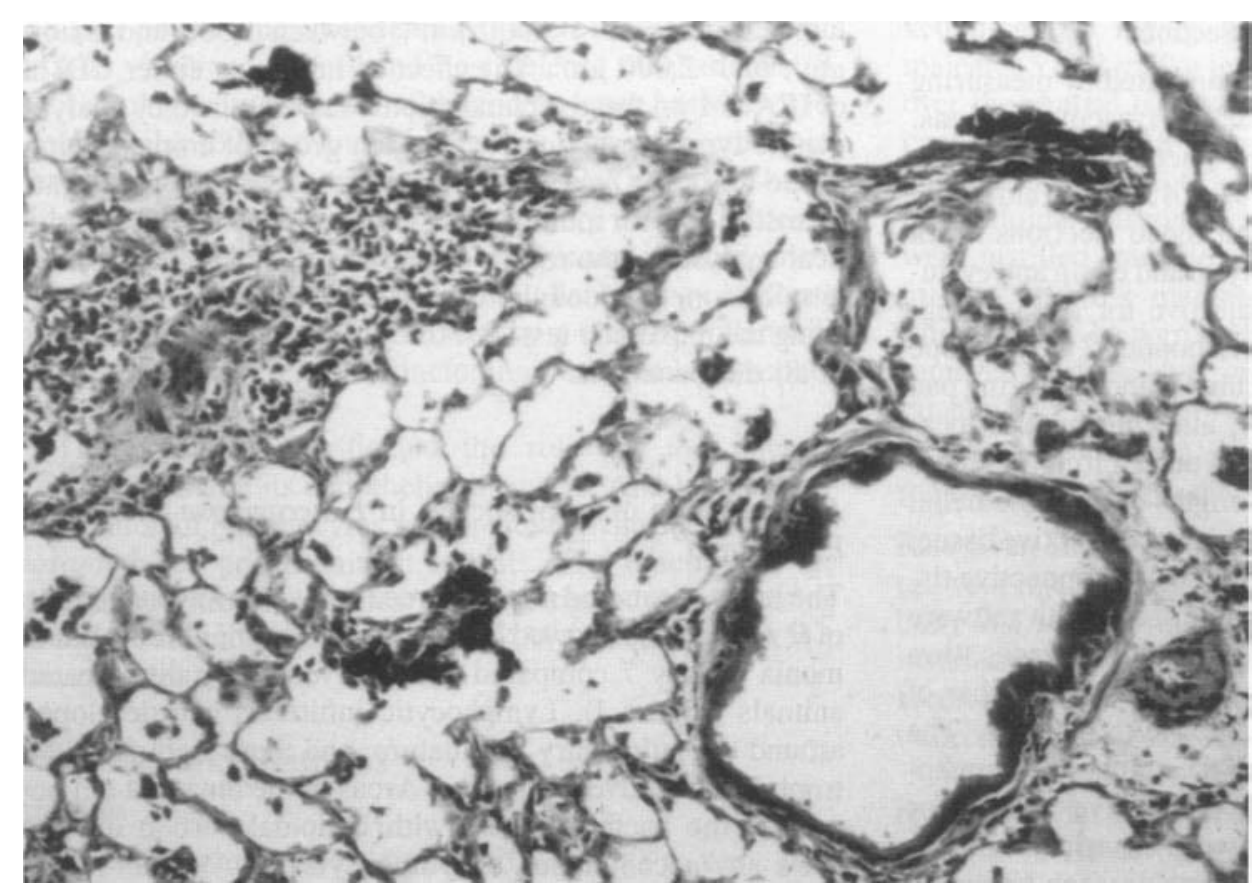

Figure 1. Histologic examination of lungs from rat 7 days after airway instillation of $P$. aeruginosa demonstrating perivascular, lymphocytic infiltration. (Original magnification: $\times 100$.)

\section{Role of CD11a and ICAM-1 in Mice}

Figure 4 shows the lymphocyte densities in perivascular connective tissue on day 7 of $P$. aeruginosa-induced pneumonia in wild-type mice. Animals received daily injections of the

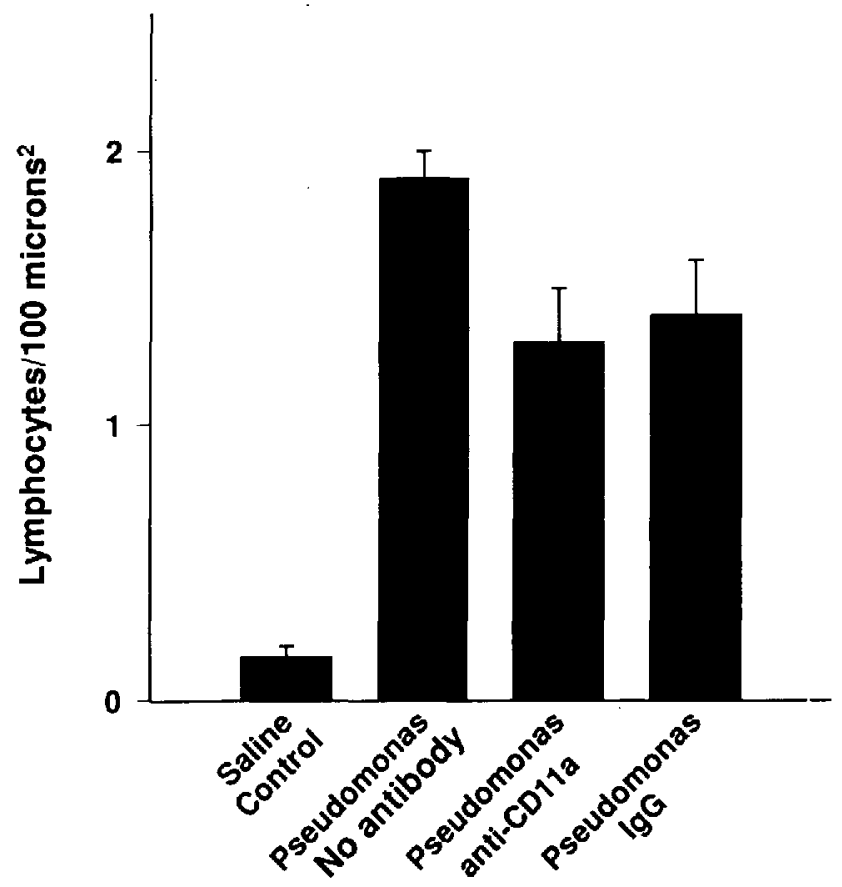

Figure 2. Role of CD1 la in lymphocyte accumulation during $P$ aentginosa-induced pneumonia in rats. Lymphocyte densities were determined from lung sections of animals that received only saline instillation in the airway compared with animals that received $P$. aeruginosa and then had daily injections of anti-CD1 la $(n=4)$, murine IgG $(n=4)$, or no antibody $(n=5)$. Data are mean \pm SEM.
anti-CD1la antibody, the anti-ICAM-1 antibody, rat IgG, or no antibody on days 3 through 6 after $P$. aeruginosa instillation. There were no significant differences in the number of lymphocytes that accumulated within the perivascular connective tissue of animals in any of the treatment groups.

Figure 5 shows the lymphocyte densities of the perivascular connective tissue grouped by vessel size for the wild-type mice treated with either anti-CD1 la, anti-ICAM-1, IgG, or no antibody. There were no significant differences in the lymphocyte accumulations between any of the treatment groups for any vessel size.

The blood lymphocyte counts on day 7 were not significantly different between wild-type mice with $P$. aerugi$n o s a$-induced pneumonia that received daily injections of either IgG or blocking antibodies to specific adhesion molecules. The anti-CD1la-treated mice had lymphocyte counts of $6.4 \pm 1.3 \times 10^{6}$ cells $/ \mathrm{ml}$ compared with $6.7 \pm$ $1.8 \times 10^{6}$ cells $/ \mathrm{ml}$ in anti-ICAM-1-treated mice and $4.2 \pm$ $0.3 \times 10^{6} \mathrm{cells} / \mathrm{ml}$ in IgG-treated mice.

Effect of ICAM-1 Deficiency on Number and Subtypes of Accumulated Lymphocytes

The lymphocyte densities in the lungs of ICAM-1 mutant and wild-type mice that received tracheal instillates of saline or $P$. aeruginosa are shown in Figure 6. There was a significant increase in the number of lymphocytes in the perivascular connective tissue in the ICAM-1 mutant mice that received $P$. aeruginosa compared with that in the ICAM-1 mutant mice that received only saline. This increase was similar to the response seen in the wild-type mice that received $P$. aeruginosa.

Analysis of immunohistochemistry done on frozen lung tissue sections did not show significant differences in the percentage of any lymphocyte subtypes in the lymphocyte infiltrates of the ICAM-1 mutant mice compared with the wild-type mice on day 7 of $P$. aeruginosa-induced pneumo- 
Figure 3. Role of CD11a in lymphocyte accumulation around different vessel sizes during $P$. aenuginosa-induced pneumonia in rats. Lymphocyte densities were determined from lung sections of animals that had $P$. aeruginosainduced pneumonia and were given daily injections of anti-CD1 la, anti-ICAM-1, murine IgG, or no antibody. Asterisk indicates significant difference compared with the no-antibody treatment group. Data are mean $\pm S E M$.

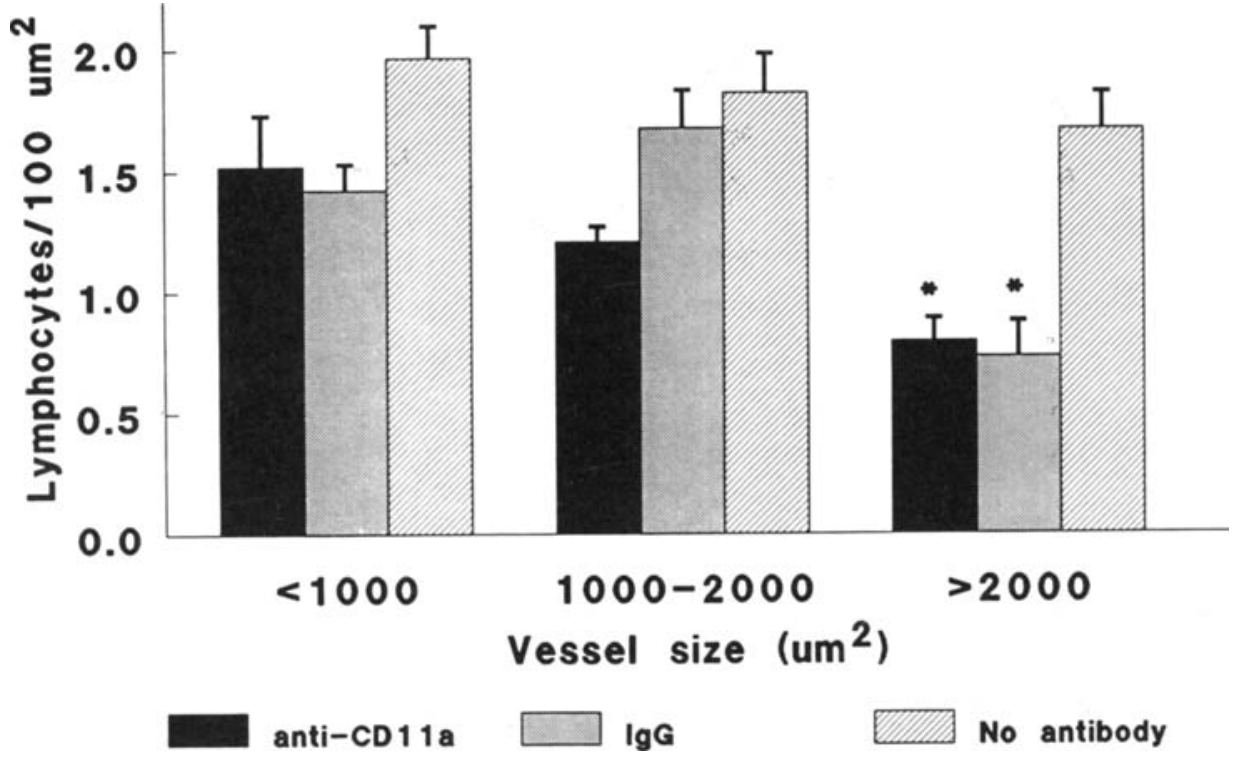

nia. The percentages of $\mathrm{CD}^{+}$cells, $\mathrm{CD8}^{+}$cells, and $\mathrm{B}$ cells were $30.5 \pm 2.2 \%, 16.1 \pm 3.2 \%$, and $19.6 \pm 5.4 \%$, respectively, in the ICAM-1 mutant mice and $37.8 \pm 2.8 \%, 22.8 \pm$ $7.7 \%$, and $23.0 \pm 2.6 \%$, respectively, in the wild-type mice.

\section{Discussion}

By 7 days after the intratracheal instillation of $P$. aeruginosa, lymphocytes accumulate in the connective tissue surrounding the microvasculature. This accumulation occurs only in the region of the lung where organisms are deposited, suggesting that lymphocytes recognize the inflamed site and accumulate in response to locally produced mediators. This study examined the roles of the adhesion molecules CD1 la and ICAM-1 in this inflammatory and immune response to $P$. aeruginosa by evaluating the lymphocyte accumulation induced by $P$. aeruginos $a$ when these adhesion molecules were inhibited or absent.

Two methods of blocking the function of these adhesion molecules were compared. Blocking monoclonal antibodies were given daily to animals with pneumonia during the period when the lymphocytic infiltrate is evolving. The accumulation of lymphocytes was morphometrically quantitated and compared with that in animals with pneumonia that received no or control antibody. In other experiments, the lymphocyte accumulation in ICAM-1 mutant mice was compared with that in wild-type mice.

TABLE 1

Circulating levels of WT.1 $24 \mathrm{~h}$ after injection evaluated using immunochemistry on cytospins from circulating leukocytes of untreated rats*

\begin{tabular}{lc}
\hline Primary Antibody & $\begin{array}{c}\text { Positive Cells } \\
(\%)\end{array}$ \\
\hline Serum samples & $38 \pm 3$ \\
WT.1 $(0.02 \mathrm{mg} / \mathrm{ml})$ & $37 \pm 4$ \\
Murine IgG & 0 \\
\hline
\end{tabular}

* Data are mean \pm SEM. $n=4$ for each primary antibody.
This study shows that in mice, the administration of blocking antibodies against either CD1 la or ICAM-1 caused no significant change in the lymphocyte accumulation during $P$. aeruginos $a$-induced pneumonia. These results suggest that neither CD1 la nor ICAM-1 are necessary for the lymphocyte accumulation to occur in this lesion. Additionally, ICAM-1 mutant mice demonstrated a significant increase in lymphocyte density in the perivascular connective tissue in re-

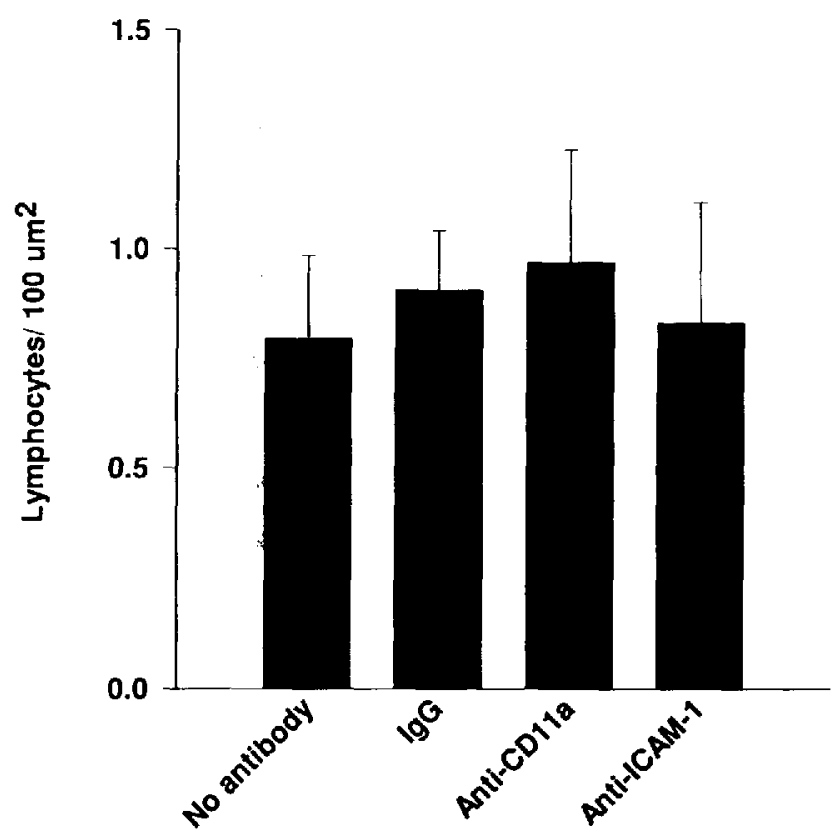

Figure 4. Role of CDI la and ICAM-1 in lymphocyte accumulation during $P$, aeruginosa-induced pneumonia in mice. Lymphocyte densities were determined from lung sections of animals with $P$. aeruginosa-induced pneumonia that received daily injections of $\mathrm{KBA}$, an anti-CD1 la antibody $(n=4) ; \mathrm{YNl} / 1.7$, an anti-ICAM-I antibody $(n=3)$; rat $\operatorname{IgG}(n=3)$; or no antibody $(n=6)$. Data are mean $\pm \mathrm{SEM}$. 


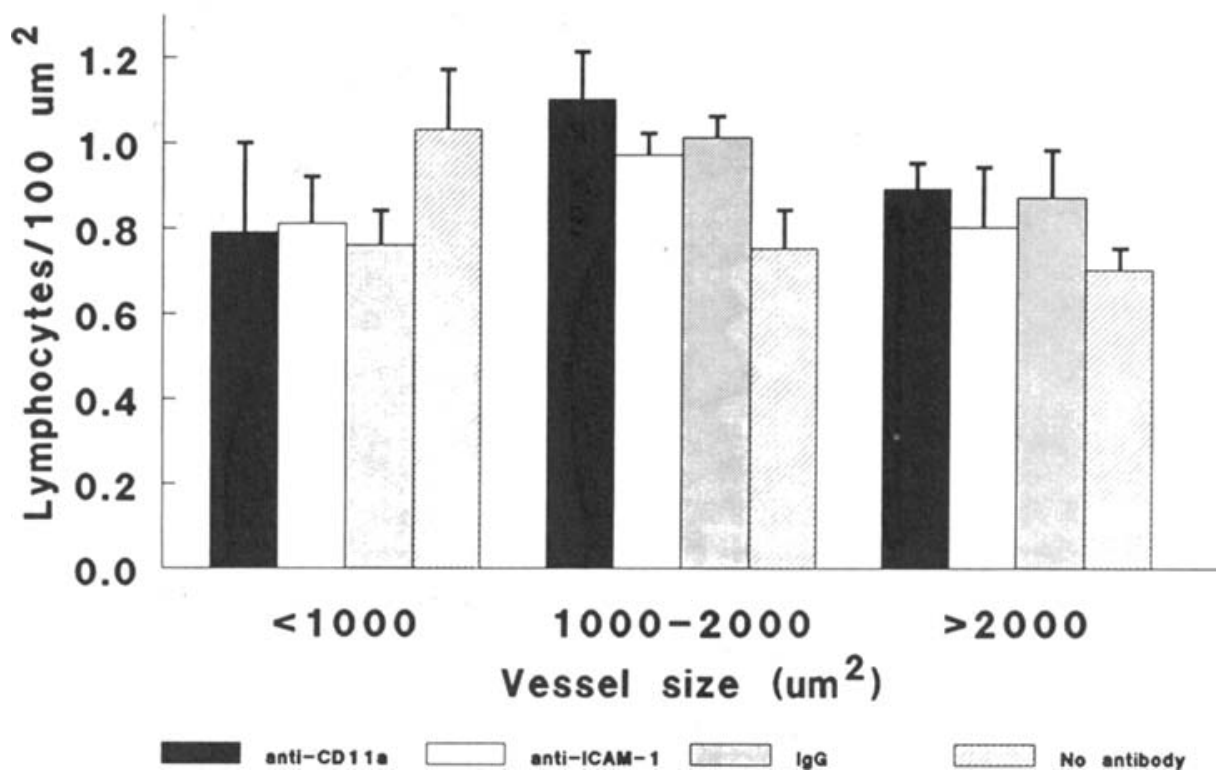

Figure 5. Role of CDIla and ICAM-1 in lymphocyte accumulation around different vessel sizes during $P$. aeruginosa-induced pneumonia in mice. Lymphocyte densities were determined from lung sections of animals that had $P$. aeruginosa-induced pneumonia and were given daily injections of anti-CD1 la, anti-ICAM-1, rat $\mathrm{IgG}$, or no antibody. Data are mean \pm SEM.

sponse to $P$. aeruginosa that was similar to that seen in wildtype mice receiving the bacteria. Further, there' were no significant differences in the subtypes of the accumulated lymphocytes in ICAM-1 mutant mice compared with that in wild-type mice. These results are consistent with the results

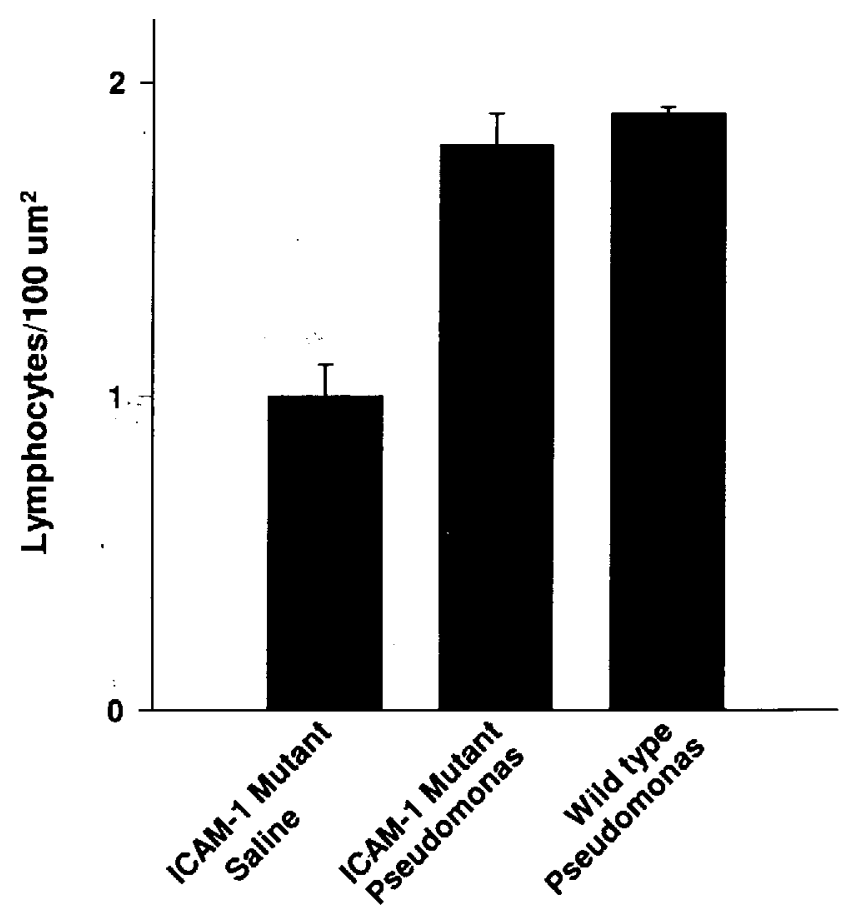

Figure 6. Effect of ICAM-1 deficiency on lymphocyte accumulation during $P$. aeruginosa-induced pneumonia in mice. Lymphocyte densities were determined from lung sections of ICAM-1 mutant mice that received saline airway instillates $(n=4)$ compared with ICAM-1 mutant mice $(n=5)$ and ICAM-1 wild-type mice $(n=4)$ with $P$. aeruginosa-induced pneumonia. Data are mean \pm SEM. using blocking antibodies, suggesting that ICAM-1 is not necessary for this lymphocyte response to occur in mice.

In rats, both the anti-CDIla antibody and the control IgG partially inhibited the lymphocyte accumulation during $P$. aeruginosa-induced pneumonia. This effect was observed only in vessels $>2,000 \mu \mathrm{m}^{2}$ in cross-sectional area and not around the smaller vessels. The partial inhibition of the lymphocyte response in both the anti-CD1 la- and the IgG-treated animals may be due to nonspecific effects of foreign proteins on cellular functions and not to inhibition of a required adhesion molecule.

Other adhesion pathways may be important in the development of this lesion. A likely candidate for this alternative pathway is the integrin very late activation antigen-4 (VLA-4) interacting with vascular cell adhesion molecule-1 (VCAM-1) (13). VLA-4, like CDIla, is expressed on the surface of memory $T$ cells (14), which are concentrated at sites of inflammation within the bronchial epithelium.

The role of adhesion molecules has been investigated in at least three other studies of chronic pulmonary inflammation. First, Piguet and associates investigated the role of CD1 1a/CD18 and CD1 lb/CD18 in chronic inflammation and interstitial fibrosis that occur following intratracheal instillation of bleomycin (15). Lymphocytes emigrate between days 3 and 14 and the infiltrate consists primarily of T cells that are $50 \%$ helper cells and 50\% suppressor cells (16-18). Pretreating mice with antibodies against CD1la and CD1lb completely prevented the bleomycin-induced increase in collagen content and the nodular and diffuse lymphocytic accumulation at 15 days (15). The neutrophil infiltrate was actually increased at late time points. In contrast to $P$. aeruginosa-induced pneumonia, these studies suggest that the natural course of bleomycin-induced injury may be delayed by blocking the function of CD1la or CD1 lb, prolonging the duration of neutrophil emigration and preventing the influx of lymphocytes and the production of collagen (15). Second, Nakajima and colleagues recently showed that blocking antibodies against either VLA-4 and VCAM-1 or CD1 la and 
ICAM-1 inhibited antigen-induced T-cell infiltration of mice tracheae (19). Third, Denis and Bisson evaluated the contribution of CD1 la to the inflammation and fibrosis seen in murine hypersensitivity pneumonitis secondary to Faeni rectivirgula (20). There were no significant differences in the number or type of cells obtained by bronchoalveolar lavage between either challenged mice given control antibody (rat IgG) and challenged mice given a rat anti-mouse CDIla monoclonal antibody. However, administration of anti-CD1 la significantly decreased the lung hydroxyproline levels and the morphometric lung index in mice with hypersensitivity pneumonitis. All these studies contrast with our study and show a role for CD1la/CD18 and ICAM-1 in the recruitment of lymphocytes in lung injury induced by bleomycin and hypersensitivity. Taken together, these studies suggest that the accumulation of lymphocytes, similar to the recruitment of neutrophils, occurs through at least two pathways, one that requires CD1 1a/ICAM-1 and one that does not. The particular stimulus and the ensuing response appear to be critical in determining which adhesion pathway is utilized.

The role of adhesion molecules in chronic inflammation in organs and tissues other than the lung has also been studied, including experiments using ICAM-1 mutant mice. Sligh and associates found that ICAM-1 mutant mice that had been sensitized and rechallenged with 2,4-dinitrofluorobenzene (DNFB) had a $74 \%$ reduction in maximal ear swelling (a measure of delayed type hypersensitivity) compared with wild-type mice (11). Histologic studies revealed an attenuation of the inflammatory cell infiltrate in the ICAM-1 mutant mice compared with the wild-type mice after rechallenge with DNFB. Although we do not yet understand the differences in the mechanisms underlying these disparate forms of chronic inflammation in the lung and other tissues, these studies underline the importance of examining lymphocyte accumulation in chronic inflammation induced by different stimuli.

Mechanisms not requiring adhesion molecules may mediate the accumulation of lymphocytes in $P$. aeruginosa-induced pneumonia. Local proliferation of lymphocytes may play a predominant role in this process. Cytokines, such as interleukin- 6 , that are produced by the endothelium and other cells at sites of inflammation could mediate this lymphocyte accumulation by stimulatory effects on cell proliferation and differentiation $(21,22)$.

In summary, these studies suggest that lymphocyte accumulation during $P$. aeruginosa-induced pneumonia in rodents occurs through mechanisms that are independent of the adhesion molecules CD1 la and ICAM-1. Either alternative adhesion pathways, perhaps involving VLA-4 and VCAM-1 (13) or L-selectin (23), or local proliferation of lymphocytes may mediate this accumulation.

Acknowledgments: This work was supported by a grant from the Riley Memorial Association to J.L.W. and PHS HL52466 to C.M.D. C.M.D. is the recipient of a Career Investigator Award from the American Lung Association. J.E.S. worked in the laboratory of Arthur L. Beaudet with support of NIH Grant AI 32177 .

\section{References}

1. Berman, J. S., D. J. Been, A. C. Theodore, H. Kornfeld, J. Bernardo, and D. M. Center. 1990. Lymphocyte recruitment to the lung. Am. Rev. Respir. Dis. 142:238-257.

2. Pabst, R. 1991. Compartmentalization and kinetics of lymphoid cells in the lung. Reg. Immunol. 3:62-71.

3. Abraham, E., A. A. Freitas, and A. A. Couthino. 1990. Purification and characterization of intraparenchymal lung lymphocytes. $J$. Immunol. $144: 2117-2122$.

4. Picker, L. J., and E. C. Butcher. 1992. Physiologic and molecular mechanisms of lymphocyte homing. Annu. Rev. Immunol. 10:561-591.

5. Stoolman, L. M. 1993. Adhesion molecules involved in leukocyte recruitment and lymphocyte recirculation. Chest 103:79S-86S.

6. Kavanaugh, A. F., E. Lightfoot, P. E. Lipsky, and N. OppenheimerMarks. 1991. Role of CD11/CD18 in adhesion and transendothelial migration of T cells. J. Immunol. 146:149-156.

7. Dustin, M. L., and T. A. Springer. 1988. Lymphocyte function-associated antigen-1 (LFA-1) interaction with intercellular adhesion molecule-1 (ICAM-1) is one of at least three mechanisms for lymphocyte adhesion to cultured endothelial cells. J. Cell Biol. 107:321-331.

8. Iwato, M., and S. Atsuhiko, 1991. Morphological and immunohistochemical studies of the lungs and bronchus-associated lymphoid tissue in a rat model of chronic pulmonary infection with Pseudomonas aeruginosa. Infect. Immun. 59:1514-1520.

9. Cash, H. A., D. E. Woods, B. McCullough, W. G. Johanson, and J. A. Bass. 1979. A rat model of chronic respiratory infection with Pseudomonas aeruginosa. Am. Rev. Respir. Dis. 119:453-459.

10. Wiebke, J. L., W. M. Quinlan, L. Graham, N. A. Doyle, and C. M. Doerschuk. 1993. Lymphocyte recruitment in response to Pseudomonas infection in the rat lung. Am. Rev. Respir. Dis. 147(Suppl.):A12. (Abstr.)

11. Sligh, J. E., C. M. Ballantyne, S. S. Rich, H. K. Hawkins, C. W. Smith, A. Bradley, and A. L. Beaudet. 1993. Inflammatory and immune responses are impaired in mice deficient in intercellular adhesion molecule 1. Proc. Natl. Acad. Sci. USA 90:8529-8533.

12. Takei, F. 1985. Inhibition of mixed lymphocyte response by a rat monoclonal antibody to a murine lymphocyte activation antigen (MALA-2). J. Immunol. 134:1403-1407.

13. Carlos, T. M. B. R. Schwartz, and N. L. Kovach. 1990 . Vascular cell adhesion molecule-1 mediates lymphocyte adherence to cytokine-activated cultured human endothelial cells. Blood 76:965-970.

14. Saltini, C., M. Kirby, B. C. Trapnell, N. Tamura, and R. G. Crystal. 1990. Biased accumulation of T-lymphocytes with memory-type CD45 leukocyte common antigen gene expression on the epithelial surface of the human lung. J. Exp. Med. 171:1123-1140.

15. Piguet, P. F., H. Rosen, C. Vesin, and G. E. Grau. 1993. Effective treatment of the pulmonary fibrosis elicited in mice by bleomycin or silica with anti-CD11 antibodies. Am. Rev. Respir. Dis. 147:435-441.

16. Snider, G. L., J. A. Hayes, and A. L. Korthy. 1978. Chronic interstitial pulmonary fibrosis produced in hamsters by endotracheal bleomycin. $\mathrm{Am}$. Rev. Respir. Dis. 117:289-297.

17. Hesterberg, T. W., J. E. Gerriets, K. M. Reiser, A. C. Jackson, C. E. Cross, and J. A. Last. 1981. Bleomycin-induced pulmonary fibrosis: correlation of biochemical, physiological, and histological changes. Toxicol. Appl. Pharmacol. 60:360-367.

18. Thrall, R. S., and R. W. Barton. 1984. A comparison of lymphocyte populations in lung tissue and in bronchoalveolar lavage fluid of rats at various times during the development of bleomycin-induced pulmonary fibrosis. Am. Rev. Respir. Dis. 129:279-283.

19. Nakajima, H., H. Sano, T. Nishimura, S. Yoshida, and I. Iwamoto. 1994. Role of vascular cell adhesion molecule-1/very late activation antigen-1 and intercellular adhesion molecule-1/ymphocyte function associated antigen-1 interactions in antigen induced eosinophil and $\mathrm{T}$ cell recruitment into the tissue. J. Exp. Med. 179:1145-1154.

20. Denis, M., and D. Bisson. 1994. Blockade of leukocyte function-associated antigen (LFA-1) in a murine model of lung inflammation. $\mathrm{Am} . J$ Respir. Cell Mol. Biol. 10:481-486.

21. Cavender, D. E. 1991. Interaction between endothelial cells and the cells of the immune system. Int. Rev. Exp. Pathol. 32:57-94.

22. Mantovani, A., F. Bussolino, and E. Dejana. 1992. Cytokine regulation of endothelial cell function. FASEB J. 6:2591-2599.

23. Spertini, O., F. W. Luscinskas, G. S. Kansas, J. M. Munro, J. D. Griffin, M. A. Gimbrone, and T. F. Tedder. 1991. Leukocyte adhesion molecule-1 (LAM-1, L-selectin) interacts with an inducible endothelial ligand to support leukocyte adhesion. J. Immunol. 147:2565-2573. 